\title{
A New Approach for Analyzing the Reliability of the Repair Facility in a Series System with Vacations
}

\author{
Renbin Liu and Yong Wu \\ School of Mathematics and Statistics, Chongqing University of Technology, \\ Chongqing 400054, China \\ Correspondence should be addressed to Renbin Liu, liurb888@126.com
}

Received 15 April 2012; Accepted 7 May 2012

Academic Editor: Francis T. K. Au

Copyright (C) 2012 R. Liu and Y. Wu. This is an open access article distributed under the Creative Commons Attribution License, which permits unrestricted use, distribution, and reproduction in any medium, provided the original work is properly cited.

\begin{abstract}
Based on the renewal process theory we develop a decomposition method to analyze the reliability of the repair facility in an $n$-unit series system with vacations. Using this approach, we study the unavailability and the mean replacement number during $(0, t]$ of the repair facility. The method proposed in this work is novel and concise, which can make us see clearly the structures of the facility indices of a series system with an unreliable repair facility, two convolution relations. Special cases and numerical examples are given to show the validity of our method.
\end{abstract}

\section{Introduction}

It is well known that the repairable systems with an unreliable repair facility are frequently studied in the literature of reliability theory. Theoretically, they are in a class of more general repairable systems, and the usual repairable systems are their special cases [1].

Common approaches in the reliability analysis of repairable systems with a repair facility include the Markov renewal process method, the geometric process method, the supplementary variable method, and the strong stability method. The Markov renewal process method has important applications in reliability theory. By means of this method, the reliability indices of various systems were obtained in the previous literatures (see, e.g., $[2,3])$. The geometric process proposed by Lam [4] is a special monotone process, which can model the working times and the repair times of a deteriorating system. Much research has been conducted by Jia and $\mathrm{Wu}[5,6], \mathrm{Wu}$ and Clements-Croome [7], Zhang and Wang [8], and others under the monotone process model. Moreover, with the help of the geometric process method, a deteriorating system with its repairman having multiple vacations was studied by 
Yuan and $\mathrm{Xu}$ [9]. They obtained the optimal replacement policy which could make the longrun expected cost per unit time minimize. The strong stability method studied by Rahmoune and Aissani [10] and other researchers is a more powerful tool. For example, Rahmoune and Aissani [10] applied this method to analyze the characteristics of the $M / G / 1 / N$ queue with multiple vacations where the rate of the vacations is sufficiently small. This method is also useful in analyzing complex repairable systems, such as, redundant repairable systems with unreliable facilities, with vacations, or with priority in use. The supplementary variable technique was first introduced by Cox [11], and it had been widely applied to complex repairable systems by Li et al. [12], Liu et al. [13, 14], and many others. Also, recently, Guo et al. [15] have derived the steady-state reliability indices of a series system by using the eigenfunction corresponding to eigenvalue 0 of the system operator.

However, these methods mentioned above usually become too complicated to be solved especially when dealing with some complex systems with many random variables following general distributions. In this paper, a new approach, called decomposition method here, is developed based on the renewal process theory. With this novel method, we analyze the unavailability and the mean replacement number during $(0, t]$ of the repair facility in an $n$-unit series system with an unreliable repair facility and vacations. To be precise, we obtain two convolution relations for the unavailability and the mean replacement number. The two convolution relations turn studying the unavailability and the mean replacement number during $(0, t]$ into studying the corresponding reliability indices in the classical oneunit system and the "generalized busy period of the repair facility" (see Definition 2.3). In addition, some new reliability results are also obtained.

To introduce our method, we consider an $n$-unit series system with a nonreliable repair facility and multiple adaptive vacations. We aim at obtaining two key reliability quantities of the repair facility, that are, the replacement probability (the unavailability of the repair facility) and the average number of replacements during $(0, t]$. Note that the two indices have been investigated by Liu et al. $[13,14]$ with the help of the supplementary variable method. In this paper we study again the above two indices by means of a decomposition method, which is completely different from the methods used in [2-15]. Our main idea is as follows: (1) with the definition of "generalized busy period of the repair facility," we obtain the probability that the repair facility is during its generalized busy period at time $t$; (2) based on this, using our decomposition method, we derive the unavailability and average replacement number of the repair facility, which lead to two convolution relations; (3) finally, we present some special cases and numerical examples to show the validity of our method.

The rest of the paper is organized as follows. The next section gives the assumptions of the considered model and some preliminaries. In Section 3 we use a decomposition method to discuss two key reliability indices of the repair facility. In Sections 4 and 5 special cases and numerical examples are presented to validate the derived results. Conclusions are finally drawn in Section 6.

\section{Assumptions and Preliminaries}

We consider a series repairable system consisting of $n$ dissimilar units, a repairman, and an unreliable repair facility by making the following assumptions.

(1) Whenever one unit fails, the system breaks down. It is assumed that $n$ units shut each other off, and each unit after repair is as good as new. As soon as the repair of the failed unit is completed, the system starts to operate immediately. 
(2) The repairman will take a random maximum number, denoted by $H$, of vacations whenever there is no failed unit waiting for repair in the system. The probability mass function of $H$ is $P(H=j)=c_{j}, j=1,2, \ldots$. The random variable $H$ may represent the maximum number tasks or jobs available for repairman to work on if the system is operating. Denote the repairman's $i$ th vacation time by $V_{i}, i \geq 1$. Assume that $V_{i}, i \geq 1$ are independent and identically distributed random variables each with the probability distribution function $V(t)$ and a mean vacation time $E(V)$. At each vacation completion instant, the repairman checks the system to see if there is a failed unit waiting and decides the action to take according to the state of the system. There are three cases: (a) if there is a failed unit waiting, the repairman will repair it immediately; (b) if there is no failed unit waiting and the total number of vacations is still less than $H$, the repairman will take another vacation; (c) if there is no failed unit waiting and the total number of vacations is equal to $H$, the repairman will stay idle and wait for the next failure.

(3) The lifetime $X_{i}$ of unit $i$ follows an exponential distribution $F_{i}(t)=1-e^{-\lambda_{i} t}, t \geq 0$, $0 \leq \lambda_{i}<\infty$. The repair time $Y_{i}$ of the failed unit $i$ obeys a general distribution function $G_{i}(t), t \geq 0$ with a mean repair time $E\left(Y_{i}\right), i=1,2, \ldots, n$.

(4) It is assumed that the repair facility neither fails nor deteriorates in its idle periods and fails only during its "generalized busy periods" presented by this paper. The life span $U$ of the repair facility has an exponential distribution $U(t)=1-e^{\alpha t}, t \geq 0$, $0 \leq \alpha<\infty$.

(5) When the repair facility fails, it is replaced by a new and identical one immediately and the unit that is being repaired has to wait for repair. The replacement time $B$ of the repair facility has an arbitrary distribution $B(t)$ with a mean replacement time $E(B)$. After replacement, the new repair facility starts its repair to the failed unit whose repair was stopped. The repair time for the failed unit is cumulative, that is, the repaired time of this failed unit is still valid.

(6) Initially, the system with $n$ new units begins to operate, the new repair facility is during its idle period, and the repairman begins to take multiple adaptive vacations. All random variables are mutually independent.

Remark 2.1. The multiple adaptive vacation policy presented by this paper is introduced by Tian and Zhang [16], which is a generalization of single vacation, multiple vacations and variant vacation, and so forth. The analysis in this work shows that our method is valid in studying the reliability indices of the repair facility of complex vacation systems with an unreliable repair facility.

For ease of reference, we list some notations and state two definitions as shown at the end of the paper.

Definition 2.2. The "generalized repair time of unit $i$ " $\tilde{Y}_{i}, i=1,2, \ldots, n$ denotes the time interval from the time when the repair facility begins to repair the failed unit $i$ until the repair of the failed unit $i$ ends, which includes some possible replacement times due to the facility failures in the process of repairing the failed unit $i$. 
For $t \geq 0$, let $\widetilde{G}_{i}(t)=P\left(\tilde{Y}_{i} \leq t\right)$, then (see $\left.[1]\right)$

$$
\widetilde{G}_{i}(t)=\sum_{k=0}^{\infty} \int_{0}^{t} B^{(k)}(t-x) \frac{(\alpha x)^{k}}{k !} e^{-\alpha x} d G_{i}(x)
$$

The Laplace-Stieltjes transform of $\widetilde{G}_{i}(t)$ is given by

$$
\tilde{g}_{i}(s)=\int_{0}^{\infty} e^{-s t} d \tilde{G}_{i}(t)=g_{i}(s+\alpha-\alpha b(s)), \quad \mathfrak{R}(s)>0,
$$

where $g_{i}(s)=\int_{0}^{\infty} e^{-s t} d G_{i}(t)$ and $b(s)=\int_{0}^{\infty} e^{-s t} d B(t)$ are the Laplace-Stieltjes transforms of $G_{i}(t)$ and $B(t)$, respectively. The mean of the generalized repaired time of the failed unit $i$ is given by

$$
E\left(\tilde{Y}_{i}\right)=-\left.\frac{d \tilde{g}_{i}(s)}{d s}\right|_{s=0}=E\left(Y_{i}\right)(1+\alpha E(B)), \quad i=1,2, \ldots, n
$$

Definition 2.3. The "generalized busy period of the repair facility" $D$ denotes the time interval from the time when the repair facility begins to repair a failed unit until the repair of the failed unit completes, where $D$ contains some possible replacement times due to the facility failures in the process of repairing the failed unit.

Remark 2.4. If the system's operating time $\min _{1 \leq i \leq n} X_{i}=X_{l}$, then $D=\tilde{Y}_{l}, l=1,2, \ldots, n$.

\section{Reliability Analysis of the Repair Facility}

In this section, by means of a decomposition method we will discuss the probability that the repair facility is during its generalized busy period at time $t$. On this basis, we study the unavailability and the replacement number during $(0, t]$ of the repair facility. Finally, we analyze the structures of the facility indices in our system.

\subsection{The Probability That the Facility Is during Its Generalized Busy Period}

For $t \geq 0$, let

$$
A(t)=P(\text { the repair facility is during its generalized busy period at time } t) \text {. }
$$

Theorem 3.1. If $\mathfrak{R}(s)>0$, then the Laplace transform of $A(t)$ is

$$
a^{*}(s)=\frac{\Theta(s)}{s\left[1-\Theta(s) \sum_{l=1}^{n}\left(\lambda_{l} / \Lambda\right) \tilde{g}_{l}(s)\right]} \sum_{l=1}^{n} \frac{\lambda_{l}}{\Lambda}\left[1-\tilde{g}_{l}(s)\right],
$$


and, in steady state, the probability that the repair facility is during its generalized busy period is given by

$$
\begin{aligned}
& \lim _{t \rightarrow \infty} A(t) \\
& =\frac{(1 / \Lambda) \sum_{i=1}^{n} \lambda_{i} E\left(Y_{i}\right)(1+\alpha E(B))}{(1 / \Lambda) \sum_{i=1}^{n} \lambda_{i} E\left(Y_{i}\right)(1+\alpha E(B))+E(V) /(1-v(\Lambda))+[1 / \Lambda-E(V) /(1-v(\Lambda))] H(v(\Lambda))},
\end{aligned}
$$

where

$$
\Theta(s)=\frac{\Lambda}{s+\Lambda} H(v(s+\Lambda))+\frac{v(s)-v(s+\Lambda)}{1-v(s+\Lambda)}[1-H(v(s+\Lambda))]
$$

Proof. Let $\Delta_{t}$ be the event that the repair facility is during its generalized busy period at time $t$. Denote $\xi_{1}$ and $\eta_{1}$ be the first operating time and the first repair time of the system, respectively. By applying the total probability decomposition technique and noting that the time points that the system begins to operate are renewal ones, we have

$$
\begin{aligned}
A(t) & =P\left(\Delta_{t}, \xi_{1}>t\right)+P\left(\Delta_{t}, \xi_{1} \leq t\right) \\
& =P\left(\Delta_{t}, \xi_{1} \leq t, \xi_{1}>\sum_{i=1}^{H} V_{i}\right)+P\left(\Delta_{t}, \xi_{1} \leq t, \xi_{1} \leq \sum_{i=1}^{H} V_{i}\right) .
\end{aligned}
$$

The first term of (3.5) can be decomposed as

$$
\begin{gathered}
\sum_{j=1}^{\infty} c_{j}\left\{P\left(\xi_{1}>\sum_{i=1}^{j} V_{i}, \xi_{1} \leq t<\xi_{1}+\eta_{1}\right)+P\left(\Delta_{t}, \xi_{1}>\sum_{i=1}^{j} V_{i}, t \geq \xi_{1}+\eta_{1}\right)\right\} \\
=\sum_{j=1}^{\infty} c_{j} \sum_{l=1}^{n}\left\{P\left(\xi_{1}=X_{l}, X_{l}>\sum_{i=1}^{j} V_{i}, X_{l} \leq t<X_{l}+\tilde{Y}_{l}\right)\right. \\
\left.+P\left(\Delta_{t}, \xi_{1}=X_{l}, X_{l}>\sum_{i=1}^{j} V_{i}, t \geq X_{l}+\tilde{Y}_{l}\right)\right\} \\
=\sum_{j=1}^{\infty} c_{j} \sum_{l=1}^{n}\left\{\int_{0}^{t}\left[1-\tilde{G}_{l}(t-x)\right] \lambda_{l} e^{-\Lambda x} V^{(j)}(x) d x\right. \\
\left.+\int_{0}^{t} \int_{0}^{t-x} A(t-x-y) d \tilde{G}_{l}(y) \lambda_{l} e^{-\Lambda x} V^{(j)}(x) d x\right\} .
\end{gathered}
$$


Similarly, we can decompose the second term of (3.5) as

$$
\begin{gathered}
\sum_{j=1}^{\infty} c_{j} \sum_{k=1}^{j}\left\{P\left(\sum_{i=1}^{k-1} V_{i} \leq \xi_{1}<\sum_{i=1}^{k} V_{i}, \sum_{i=1}^{k} V_{i}<t \leq \sum_{i=1}^{k} V_{i}+\eta_{1}\right)\right. \\
\left.+P\left(\Delta_{t}, \sum_{i=1}^{k-1} V_{i} \leq \xi_{1}<\sum_{i=1}^{k} V_{i}, t \geq \sum_{i=1}^{k} V_{i}+\eta_{1}\right)\right\} \\
=\sum_{j=1}^{\infty} c_{j} \sum_{k=1}^{j} \sum_{l=1}^{n}\left\{P\left(\xi_{1}=X_{l}, \sum_{i=1}^{k-1} V_{i} \leq X_{l}<\sum_{i=1}^{k} V_{i}, \sum_{i=1}^{k} V_{i}<t \leq \sum_{i=1}^{k} V_{i}+\tilde{Y}_{l}\right)\right. \\
\left.+P\left(\Delta_{t}, \xi_{1}=X_{l}, \sum_{i=1}^{k-1} V_{i} \leq X_{l}<\sum_{i=1}^{k} V_{i}, t \geq \sum_{i=1}^{k} V_{i}+\tilde{Y}_{l}\right)\right\} \\
=\sum_{j=1}^{\infty} c_{j} \sum_{k=1}^{j} \sum_{l=1}^{n} \frac{\lambda_{l}}{\Lambda}\left\{\int_{0}^{t} \int_{0}^{t-x}\left[1-\tilde{G}_{l}(t-x-u)\right]\left[1-e^{-\Lambda u}\right] d V(u) e^{-\Lambda x} d V^{(k-1)}(x)\right. \\
+\int_{0}^{t} \int_{0}^{t-x} \int_{0}^{t-x-u} A(t-x-u-y) d \tilde{G}_{l}(y) \\
\left.\times\left[1-e^{-\Lambda u}\right] d V(u) e^{-\Lambda x} d V^{(k-1)}(x)\right\}
\end{gathered}
$$

Taking the Laplace transforms of (3.5)-(3.7) and simplifying lead to

$$
\begin{array}{r}
a^{*}(s)=\sum_{j=1}^{\infty} c_{j} \sum_{l=1}^{n}\left\{\frac{\lambda_{l}\left(1-\tilde{g}_{l}(s)\right)}{s(s+\Lambda)}[v(s+\Lambda)]^{j}+\frac{\lambda_{l} a^{*}(s) \tilde{g}_{l}(s)}{s+\Lambda}[v(s+\Lambda)]^{j}\right\} \\
+\sum_{j=1}^{\infty} c_{j} \sum_{k=1}^{j} \sum_{l=1}^{n} \frac{\lambda_{l}}{\Lambda}\left\{\frac{1-\widetilde{g}_{l}(s)}{s}[v(s+\Lambda)]^{k-1}[v(s)-v(s+\Lambda)]\right. \\
\left.+a^{*}(s) \tilde{g}_{l}(s)[v(s)-v(s+\Lambda)][v(s+\Lambda)]^{k-1}\right\} .
\end{array}
$$

Solving (3.8), we can get (3.2). Applying the well-known Tauberian theorem [17], we have

$$
\lim _{t \rightarrow \infty} A(t)=\lim _{s \rightarrow 0} s a^{*}(s) .
$$

Putting (3.2) into the above equation and using L' Hospital's rule complete the proof of Theorem 3.1.

To study the unavailability and the replacement number during $(0, t]$ of the repair facility, we first consider a classical one-unit replaceable system $[18,19]$. When the unit fails, it is replaced by a new and identical one immediately. The lifetime $U$ of the unit has an exponential distribution $U(t)=1-e^{-\alpha t}(t \geq 0,0 \leq \alpha<\infty)$, and the replacement time $B$ of 
the failed unit obeys an arbitrary distribution $B(t)$ with a mean replacement time $E(B) . U$ and $B$ are mutually independent. After replacement, the new unit begins to operate immediately. For $t \geq 0$, let

$$
\begin{gathered}
\Phi_{0}(t)=P(\text { the unit is being replaced at time } t), \\
M_{0}(t)=E(\text { the replacement number of the unit during }(0, t]),
\end{gathered}
$$

and $\varphi_{0}^{*}(s)=\int_{0}^{\infty} e^{-s t} \Phi_{0}(t) d t, m_{0}(s)=\int_{0}^{\infty} e^{-s t} d M_{0}(t)$.

Lemma 3.2 (see $[18,19])$. If $\Re(s)>0$, then

$$
\begin{gathered}
\varphi_{0}^{*}(s)=\frac{\alpha[1-b(s)]}{s[s+\alpha-\alpha b(s)]}, \quad m_{0}(s)=\frac{\alpha}{s+\alpha-\alpha b(s)}, \\
\lim _{t \rightarrow \infty} \Phi_{0}(t)=\lim _{s \rightarrow 0} s \varphi_{0}^{*}(s)=\frac{\alpha E(B)}{1+\alpha E(B)}, \quad \lim _{t \rightarrow \infty} \frac{M_{0}(t)}{t}=\lim _{s \rightarrow 0} s m_{0}(s)=\frac{\alpha}{1+\alpha E(B)} .
\end{gathered}
$$

\subsection{The Unavailability of the Repair Facility}

Now, we discuss the unavailability of the repair facility at time $t$, that is, the probability that the repair facility is being replaced at time $t$. For $t \geq 0$, let

$$
\Phi(t)=P(\text { the repair facility is being replaced at time } t) .
$$

Theorem 3.3. Let $\varphi^{*}(s)=\int_{0}^{\infty} e^{-s t} \Phi(t) d t$. If $\Re(s)>0$, then

$$
\varphi^{*}(s)=\varphi_{0}^{*}(s)\left[s a^{*}(s)\right]
$$

and the steady-state unavailability of the repair facility is given by

$$
\begin{aligned}
& \lim _{t \rightarrow \infty} \Phi(t) \\
& =\frac{(\alpha / \Lambda) \sum_{l=1}^{n} \lambda_{l} E\left(Y_{l}\right) E(B)}{(1 / \Lambda) \sum_{l=1}^{n} \lambda_{l} E\left(Y_{l}\right)(1+\alpha E(B))+E(V) /(1-v(\Lambda))+[1 / \Lambda-E(V) /(1-v(\Lambda))] H(v(\Lambda))},
\end{aligned}
$$

where $\varphi_{0}^{*}(s)$ and $a^{*}(s)$ are given by Lemma 3.2 and (3.2), respectively.

Proof. (i) In the light of the assumptions, the repair facility neither fails nor deteriorates in its idle period and it is available at the beginning time as well as the ending time of each generalized busy period, then the repair facility is replaced at time $t$ if and only if the time $t$ is during one generalized busy period of the repair facility, and the repair facility is replaced at time $t$. Therefore, according to total probability decomposition and the renewal point 
technique, $\Phi(t)$ is decomposed as

$$
\begin{aligned}
& \Phi(t)=P\left(\text { the repair facility is being replaced at time } t, \xi_{1}>\sum_{i=1}^{H} V_{i}, \xi_{1} \leq t\right) \\
& +P\left(\text { the repair facility is being replaced at time } t, \xi_{1} \leq \sum_{i=1}^{H} V_{i}, \xi_{1} \leq t\right) \\
& =\sum_{j=1}^{\infty} c_{j} \sum_{l=1}^{n}\left\{P \left(\text { the repair facility is being replaced at time } t, \xi_{1}=X_{l}\right.\right. \text {, } \\
& \left.X_{l}>\sum_{i=1}^{j} V_{i}, X_{l} \leq t<X_{l}+\tilde{Y}_{l}\right) \\
& +P(\text { the facility is being replaced at time } t \text {, } \\
& \left.\left.\xi_{1}=X_{l}, X_{l}>\sum_{i=1}^{j} V_{i}, X_{l}+\tilde{Y}_{l} \leq t\right)\right\} \\
& +\sum_{j=1}^{\infty} c_{j} \sum_{k=1}^{j} \sum_{l=1}^{n}\left\{P \left(\text { the repair facility is being replaced at time } t, \xi_{1}=X_{l},\right.\right. \\
& \left.\sum_{i=1}^{k-1} V_{i} \leq X_{l}<\sum_{i=1}^{k} V_{i}, \sum_{i=1}^{k} V_{i} \leq t<\sum_{i=1}^{k} V_{i}+\tilde{Y}_{l}\right) \\
& +P\left(\text { the repair facility is being replaced at time } t, \xi_{1}=X_{l}\right. \text {, } \\
& \left.\left.\sum_{i=1}^{k-1} V_{i} \leq X_{l}<\sum_{i=1}^{k} V_{i}, \sum_{i=1}^{k} V_{i}+\tilde{Y}_{l} \leq t\right)\right\} \\
& =\sum_{j=1}^{\infty} c_{j} \sum_{l=1}^{n}\left\{\int_{0}^{t} S_{l}(t-x) \lambda_{l} e^{-\Lambda x} V^{(j)}(x) d x\right. \\
& \left.+\int_{0}^{t} \int_{0}^{t-x} \Phi(t-x-y) d \tilde{G}_{l}(y) \lambda_{l} e^{-\Lambda x} V^{(j)}(x) d x\right\} \\
& +\sum_{j=1}^{\infty} c_{j} \sum_{k=1}^{j} \sum_{l=1}^{n} \frac{\lambda_{l}}{\Lambda}\left\{\int_{0}^{t} \int_{0}^{t-x} S_{l}(t-x-u)\left[1-e^{-\Lambda u}\right] d V(u) e^{-\Lambda x} d V^{(k-1)}(x)\right. \\
& +\int_{0}^{t} \int_{0}^{t-x} \int_{0}^{t-x-u} \Phi(t-x-u-y) d \tilde{G}_{l}(y) \\
& \left.\times\left[1-e^{-\Lambda u}\right] d V(u) e^{-\Lambda x} d V^{(k-1)}(x)\right\}
\end{aligned}
$$

where $S_{l}(t)=P\left(D=\tilde{Y}_{l}>t \geq 0\right.$; the repair facility is being replaced at time $\left.t\right)$. 
(ii) For $t \geq 0$,

$$
S_{l}(t)=\Phi_{0}(t)-\int_{0}^{t} \Phi_{0}(t-x) d \widetilde{G}_{l}(x)
$$

where $\Phi_{0}(t)$ is determined by Lemma 3.2.

In fact, in the generalized busy period the repair facility may be in one of two states: operation or replacement, and it is available at the beginning and ending times of each generalized busy period. According to the fact that the life span of the repair facility has an exponential distribution, and conditioning on the generalized busy period, we have the decomposition of $\Phi_{0}(t)$ as follows:

$$
\begin{aligned}
\Phi_{0}(t)= & P\left(\text { the repair facility is being replaced at time } t ; D=\tilde{Y}_{l} \leq t\right) \\
& +P\left(\text { the repair facility is being replaced at time } t ; D=\tilde{Y}_{l}>t\right) \\
= & \int_{0}^{t} P(\text { the repair facility is being replaced at time } t-x \mid D=x) d \tilde{G}_{l}(x)+S_{l}(t) \\
= & \int_{0}^{t} \Phi_{0}(t-x) d \tilde{G}_{l}(x)+S_{l}(t) .
\end{aligned}
$$

So (3.16) holds.

(iii) Substituting (3.16) into (3.15) and making the Laplace transform we get (3.13). Equation (3.14) is obtained by the Tauberian theorem, Lemma 3.2 and (3.3).

\subsection{The Replacement Number of the Repair Facility}

For $t \geq 0$, let

$$
M(t)=E(\text { the replacement number of the repair facility during }(0, t]) .
$$

Theorem 3.4. Let $m(s)=\int_{0}^{\infty} e^{-s t} d M(t)$. If $\Re(s)>0$, then

$$
m(s)=m_{0}(s)\left[s a^{*}(s)\right],
$$

and the steady-state replacement frequency of the repair facility is

$$
\begin{aligned}
& \lim _{t \rightarrow \infty} \frac{M(t)}{t} \\
& =\frac{(\alpha / \Lambda) \sum_{l=1}^{n} \lambda_{l} E\left(Y_{l}\right)}{(1 / \Lambda) \sum_{l=1}^{n} \lambda_{l} E\left(Y_{l}\right)(1+\alpha E(B))+E(V) /(1-v(\Lambda))+[(1 / \Lambda)-E(V) /(1-v(\Lambda))] H(v(\Lambda))} .
\end{aligned}
$$


Proof. (1) For $t \geq 0$, let

$$
\begin{aligned}
& I_{l}(t)=E\left(0 \leq t<\tilde{Y}_{l} ; \text { the replacement number of the repair facility during }(0, t]\right), \\
& J_{l}(t)=E\left(\tilde{Y}_{l} \leq t ; \text { the replacement number of the repair facility during }\left(0, \tilde{Y}_{l}\right]\right),
\end{aligned}
$$

then similar to (3.16) above, by conditioning on the generalized busy period we have

$$
I_{l}(t)+J_{l}(t)=M_{0}(t)-\int_{0}^{t} M_{0}(t-x) d \tilde{G}_{l}(x),
$$

where $M_{0}(t)$ is determined by Lemma 3.2.

(2) Using total probability decomposition, we have the decomposition of $M(t)$ as follows:

$$
\begin{gathered}
M(t)=\sum_{j=1}^{\infty} c_{j} \sum_{k=1}^{j} \sum_{l=1}^{n} E(\text { the replacement number of the facility during }(0, t], \\
\left.\xi_{1}=X_{l}, \sum_{i=1}^{k-1} V_{i}<X_{l} \leq \sum_{i=1}^{k} V_{i}, \sum_{i=1}^{k} V_{i} \leq t<\sum_{i=1}^{k} V_{i}+\tilde{Y}_{l}\right) \\
+\sum_{j=1}^{\infty} c_{j} \sum_{l=1}^{n} E(\text { the replacement number of the facility during }(0, t], \\
+\sum_{j=1}^{\infty} c_{j} \sum_{k=1}^{j} \sum_{l=1}^{n} E(\text { the replacement number of the facility during }(0, t], \\
\left.\xi_{l}, X_{l}>\sum_{i=1}^{j} V_{i}, X_{l} \leq t<X_{l}+\tilde{Y}_{l}\right) \\
+\sum_{j=1}^{\infty} c_{j} \sum_{l=1}^{n} E(\text { the replacement number of the facility during }(0, t], \\
\left.\xi_{1}=X_{l}, X_{l}>\sum_{i=1}^{j} V_{i}, t \geq X_{l}+\tilde{Y}_{l}\right) .
\end{gathered}
$$


Based on the definition of $I_{l}(t)$ and the renewal point technique, the sum of the first and second terms of (3.23) gives

$$
\begin{gathered}
\sum_{j=1}^{\infty} c_{j} \sum_{k=1}^{j} \sum_{l=1}^{n} \frac{\lambda_{l}}{\Lambda} \int_{0}^{t} \int_{0}^{t-x} I_{l}(t-x-u)\left(1-e^{-\Lambda u}\right) d V(u) e^{-\Lambda x} d V^{(k-1)}(x) \\
+\sum_{j=1}^{\infty} c_{j} \sum_{l=1}^{n} \int_{0}^{t} I_{l}(t-x) \lambda_{l} e^{-\Lambda x} V^{(j)}(x) d x .
\end{gathered}
$$

Similarly, by the definitions of $J_{l}(t)$ and $M(t)$, the sum of the third and fourth terms of (3.23) is decomposed as

$$
\begin{aligned}
& \sum_{j=1}^{\infty} c_{j} \sum_{k=1}^{j} \sum_{l=1}^{n}\left\{E \left(\text { the replacement number of the facility during }\left(0, \sum_{i=1}^{k} V_{i}+\tilde{Y}_{l}\right]\right.\right. \text {, } \\
& \left.\xi_{1}=X_{l}, \sum_{i=1}^{k-1} V_{i}<X_{l} \leq \sum_{i=1}^{k} V_{i}, t \geq \sum_{i=1}^{k} V_{i}+\tilde{Y}_{l}\right) \\
& +E \text { (the replacement number of the facility during }\left(\sum_{i=1}^{k} V_{i}+\tilde{Y}_{l}, t\right] \text {, } \\
& \left.\left.\xi_{1}=X_{l}, \sum_{i=1}^{k-1} V_{i}<X_{l} \leq \sum_{i=1}^{k} V_{i}, t \geq \sum_{i=1}^{k} V_{i}+\tilde{Y}_{l}\right)\right\} \\
& +\sum_{j=1}^{\infty} c_{j} \sum_{l=1}^{n}\left\{E \left(\text { the replacement number of the facility during }\left(0, X_{l}+\tilde{Y}_{l}\right]\right.\right. \text {, } \\
& \left.\xi_{1}=X_{l}, X_{l}>\sum_{i=1}^{j} V_{i}, t \geq X_{l}+\tilde{Y}_{l}\right), \\
& +E\left(\text { the replacement number of the facility during }\left(X_{l}+\tilde{Y}_{l}, t\right]\right. \text {, } \\
& \left.\left.\xi_{1}=X_{l}, X_{l}>\sum_{i=1}^{j} V_{i}, t \geq X_{l}+\tilde{Y}_{l}\right)\right\} \\
& =\sum_{j=1}^{\infty} c_{j} \sum_{k=1}^{j} \sum_{l=1}^{n} \frac{\lambda_{l}}{\Lambda}\left\{\int_{0}^{t-x} J_{l}(t-x-u)\left(1-e^{-\Lambda u}\right) d V(u) e^{-\Lambda x} d V^{(k-1)}(x)\right. \\
& +\int_{0}^{t} \int_{0}^{t-x} \int_{0}^{t-x-u} M(t-x-u-y) d \widetilde{G}_{l}(y)\left(1-e^{-\Lambda u}\right) \\
& \left.\times d V(u) e^{-\Lambda x} d V^{(k-1)}(x)\right\}
\end{aligned}
$$




$$
\begin{aligned}
+\sum_{j=1}^{\infty} c_{j} \sum_{l=1}^{n}\left\{\int_{0}^{t} J_{l}(t-x) \lambda_{l} e^{-\Lambda x} V^{(j)}(x) d x\right. \\
\left.+\int_{0}^{t} \int_{0}^{t-x} M(t-x-y) d \tilde{G}_{l}(y) \lambda_{l} e^{-\Lambda x} V^{(j)}(x) d x\right\} .
\end{aligned}
$$

Inserting (3.24), (3.25), and (3.22) in (3.23), taking the Laplace-Stieltjes transform of (3.23) and simplifying lead to

$$
\begin{gathered}
m(s)=\sum_{j=1}^{\infty} c_{j} \sum_{k=1}^{j} \sum_{l=1}^{n} \frac{\lambda_{l}}{\Lambda}\left\{m(s) \tilde{g}_{l}(s)[v(s)-v(s+\Lambda)][v(s+\Lambda)]^{k-1}\right. \\
\left.+m_{0}(s)\left[1-\tilde{g}_{l}(s)\right][v(s)-v(s+\Lambda)][v(s+\Lambda)]^{k-1}\right\} \\
+\sum_{j=1}^{\infty} c_{j} \sum_{l=1}^{n}\left\{m_{0}(s)\left[1-\widetilde{g}_{l}(s)\right] \frac{\lambda_{l}}{s+\Lambda}[v(s+\Lambda)]^{j}\right. \\
\left.+m(s) \widetilde{g}_{l}(s) \frac{\lambda_{l}}{s+\Lambda}[v(s+\Lambda)]^{j}\right\} .
\end{gathered}
$$

Solving (3.26) and using (3.2) we get (3.19). By the Tauberian theorem, we have

$$
\lim _{t \rightarrow \infty} \frac{M(t)}{t}=\lim _{s \rightarrow 0} s m(s)
$$

Substituting (3.19) into the above equation and applying Lemma 3.2 and (3.3) give (3.20).

Remark 3.5. Taking the Laplace and Laplace-Stieltjes inverse transforms of (3.13) and (3.19), respectively, we obtain two convolution relations as follows:

$$
\begin{gathered}
\Phi(t)=\Phi_{0}(t) * A(t)=\int_{0}^{t} \Phi_{0}(t-x) d A(x), \quad t \geq 0, \\
M(t)=M_{0}(t) * A(t)=\int_{0}^{t} M_{0}(t-x) d A(x), \quad t \geq 0 .
\end{gathered}
$$

Clearly, (3.28) turn studying the unavailability and the replacement number during $(0, t]$ of the repair facility into studying the corresponding indices in the classical one-unit system and the probability that the repair facility is during its generalized busy period presented in this paper. It is important that the above results are not derived by the supplementary variable method in $[13,14]$.

Remark 3.6. It is seen from (3.3), (3.14), and (3.20), that the steady-state relation equations $\lim _{t \rightarrow \infty} \Phi(t)=\lim _{t \rightarrow \infty} \Phi_{0}(t) \lim _{t \rightarrow \infty} A(t)$ and $\lim _{t \rightarrow \infty}(M(t) / t)=\lim _{t \rightarrow \infty}\left(M_{0}(t) / t\right) \lim _{t \rightarrow \infty} A(t)$ also hold. The two steady-state relations are also new, which are not obtained in $[13,14]$. 
Remark 3.7. By the decomposition method used in this paper, we can also analyze the reliability indices of the series system presented by this paper.

\section{Special Cases}

Case 1. If $H=\infty, H(z)=0$, that is, the repairman in the system takes multiple vacations, then (3.3), (3.14), and (3.20), can be simplified as

$$
\begin{gathered}
\lim _{t \rightarrow \infty} A(t)=\frac{1}{\delta \Lambda} \sum_{l=1}^{n} \lambda_{l} E\left(Y_{l}\right)(1+\alpha E(B)), \quad \lim _{t \rightarrow \infty} \Phi(t)=\frac{\alpha}{\delta \Lambda} \sum_{l=1}^{n} \lambda_{l} E\left(Y_{l}\right) E(B), \\
\lim _{t \rightarrow \infty} \frac{M(t)}{t}=\frac{\alpha}{\delta \Lambda} \sum_{l=1}^{n} \lambda_{l} E\left(Y_{l}\right),
\end{gathered}
$$

where $\delta=(1 / \Lambda) \sum_{l=1}^{n} \lambda_{l} E\left(Y_{l}\right)(1+\alpha E(B))+E(V) /(1-v(\Lambda))$.

In this case, the steady-state unavailability $\lim _{t \rightarrow \infty} \Phi(t)$ and steady-state replacement frequency $\lim _{t \rightarrow \infty}((M(t)) / t)$ are identical to those in [13], which are analyzed with the help of the supplementary variable method. Also, the steady-state probability $\lim _{t \rightarrow \infty} A(t)$ is not obtained in [13].

Case 2. If $H=1, H(z)=z$, that is, the repairman in the system takes a single vacation, then

$$
\begin{gathered}
\lim _{t \rightarrow \infty} A(t)=\frac{1}{\delta \Lambda} \sum_{l=1}^{n} \lambda_{l} E\left(Y_{l}\right)(1+\alpha E(B)), \quad \lim _{t \rightarrow \infty} \Phi(t)=\frac{\alpha}{\delta \Lambda} \sum_{l=1}^{n} \lambda_{l} E\left(Y_{l}\right) E(B), \\
\lim _{t \rightarrow \infty} \frac{M(t)}{t}=\frac{\alpha}{\delta \Lambda} \sum_{l=1}^{n} \lambda_{l} E\left(Y_{l}\right),
\end{gathered}
$$

where $\delta=(1 / \Lambda) \sum_{l=1}^{n} \lambda_{l} E\left(Y_{l}\right)(1+\alpha E(B))+E(V)+(1 / \Lambda) v(\Lambda)$.

In this case, $\lim _{t \rightarrow \infty} \Phi(t)$ and $\lim _{t \rightarrow \infty}(M(t) / t)$ are in accordance with the results obtained by Liu et al. [14], which are discussed by means of the supplementary variable method. In addition, $\lim _{t \rightarrow \infty} A(t)$ is a new result.

Case 3. If $H=1, H(z)=z$ and $V(t)=1-e^{-r t}, t \geq 0$, then letting $r \rightarrow \infty$, we get the following indices of the repair facility of the series system without vacation:

$$
\begin{gathered}
\lim _{t \rightarrow \infty} A(t)=\frac{1}{\delta \Lambda} \sum_{l=1}^{n} \lambda_{l} E\left(Y_{l}\right)(1+\alpha E(B)), \quad \lim _{t \rightarrow \infty} \Phi(t)=\frac{\alpha}{\delta \Lambda} \sum_{l=1}^{n} \lambda_{l} E\left(Y_{l}\right) E(B), \\
\lim _{t \rightarrow \infty} \frac{M(t)}{t}=\frac{\alpha}{\delta \Lambda} \sum_{l=1}^{n} \lambda_{l} E\left(Y_{l}\right),
\end{gathered}
$$

where $\delta=(1 / \Lambda) \sum_{l=1}^{n} \lambda_{l} E\left(Y_{l}\right)(1+\alpha E(B))+(1 / \Lambda)$.

In this special case, $\lim _{t \rightarrow \infty} \Phi(t)$ and $\lim _{t \rightarrow \infty}(M(t) / t)$ coincide with the results obtained in [13] or [14], which are derived using the supplementary variable method. 
Table 1: Reliability indices for varying value of $E(Y)$ with $(\alpha, E(B))=(0.2,0.6)$.

\begin{tabular}{lccc}
\hline$E(Y)$ & $\lim _{t \rightarrow \infty} A(t)$ & $\lim _{t \rightarrow \infty} \Phi(t)$ & $\lim _{t \rightarrow \infty}(M(t)) / t$ \\
\hline 0.4 & 0.1650 & 0.0177 & 0.0295 \\
0.5 & 0.1981 & 0.0212 & 0.0354 \\
0.6 & 0.2287 & 0.0245 & 0.0408 \\
0.7 & 0.2570 & 0.0275 & 0.0459 \\
0.8 & 0.2833 & 0.0304 & 0.0506 \\
\hline
\end{tabular}

Table 2: Reliability indices for varying value of $\alpha$ with $(E(Y), E(B))=(0.5,0.6)$.

\begin{tabular}{lccc}
\hline$\alpha$ & $\lim _{t \rightarrow \infty} A(t)$ & $\lim _{t \rightarrow \infty} \Phi(t)$ & $\lim _{t \rightarrow \infty}(M(t)) / t$ \\
\hline 0.1 & 0.1895 & 0.0107 & 0.0179 \\
0.2 & 0.1981 & 0.0212 & 0.0354 \\
0.3 & 0.2065 & 0.0315 & 0.0525 \\
0.4 & 0.2148 & 0.0416 & 0.0693 \\
0.5 & 0.2229 & 0.0514 & 0.0857 \\
\hline
\end{tabular}

Table 3: Reliability indices for varying value of $E(B)$ with $(E(Y), \alpha)=(0.5,0.2)$.

\begin{tabular}{lccc}
\hline$E(B)$ & $\lim _{t \rightarrow \infty} A(t)$ & $\lim _{t \rightarrow \infty} \Phi(t)$ & $\lim _{t \rightarrow \infty}(M(t)) / t$ \\
\hline 0.2 & 0.1866 & 0.0072 & 0.0359 \\
0.4 & 0.1924 & 0.0143 & 0.0356 \\
0.6 & 0.1981 & 0.0212 & 0.0354 \\
0.8 & 0.2037 & 0.0281 & 0.0351 \\
1.0 & 0.2093 & 0.0349 & 0.0349 \\
\hline
\end{tabular}

\section{Numerical Examples}

In this section, some numerical examples are presented to analyze the effects of various parameters on the derived steady-state results, including the probability that the repair facility is during its generalized busy period $\lim _{t \rightarrow \infty} A(t)$, the unreliability $\lim _{t \rightarrow \infty} \Phi(t)$ and the replacement frequency $\lim _{t \rightarrow \infty}((M(t)) / t)$. For convenience, we consider an $n$-identicalcomponent series system with a nonreliable repair facility and multiple adaptive vacations. Let

(1) $n=3, \lambda_{1}=\lambda_{2}=\lambda_{3}=0.3, E\left(Y_{1}\right)=E\left(Y_{2}\right)=E\left(Y_{3}\right)=E(Y)$;

(2) $H$ follows a geometric distribution with probability generating function $H(z)=$ $0.4 z /(1-0.6 z),|z| \leq 1$

(3) $V$ obeys an exponential distribution with a mean $E(V)=1$.

Numerical results are reported in Tables $1-3$. Table 1 shows that the effects of varying mean repair time $E(Y)$ on the reliability indices of the facility for the set of parameters $(\alpha, E(B))=(0.2,0.6)$. We observe that the probability $\lim _{t \rightarrow \infty} A(t)$, the unreliability $\lim _{t \rightarrow \infty} \Phi(t)$ and the replacement frequency $\lim _{t \rightarrow \infty}(M(t) / t)$ all increase monotonously as the value $E(Y)$ increases. The effects of varying failure rate $\alpha$ on the reliability indices of the facility are shown in Table 2, where we set $(E(Y), E(B))=(0.5,0.6)$. As is to be expected, the probability $\lim _{t \rightarrow \infty} A(t)$, the unreliability $\lim _{t \rightarrow \infty} \Phi(t)$, and the replacement frequency $\lim _{t \rightarrow \infty}((M(t)) / t)$ all decrease monotonously as the rate $\alpha$ decreases. Table 3 reports the effects of varying mean replacement time $E(B)$ on the reliability indices of the facility for the 
set of parameters $(E(Y), \alpha)=(0.5,0.2)$. We note that when $E(B)$ increases, both $\lim _{t \rightarrow \infty} A(t)$ and $\lim _{t \rightarrow \infty} \Phi(t)$ increase, while $\lim _{t \rightarrow \infty}(M(t) / t)$ decreases. The trends shown by the tables are as expected.

\section{Conclusions}

In this paper, based on the renewal process theory we develop a new approach-the decomposition method. According to the method, we analyze the unavailability and average replacement number during $(0, t]$ of the repair facility in a series vacation system with an unreliable repair facility. On this basis, we derive two convolution relations, which make us see clearly the structures of the facility indices in our system. Special cases and numerical examples show that our method is valid in studying the reliability indices of the repair facility of complex vacation systems with a nonreliable repair facility.

\section{Notations}

$g(s): \quad$ The Laplace-Stieltjes transform of $G(t)$, that is, $g(s)=\int_{0}^{\infty} e^{-s t} d G(t)$

$g^{*}(s): \quad$ The Laplace transform of $G(t)$, that is, $g^{*}(s)=\int_{0}^{\infty} e^{-s t} G(t) d t$

$G^{(k)}(t): \quad$ The $k$-fold convolution of $G(t)$ with itself $(k \geq 1)$ and $G^{(0)}(t)=1, t \geq 0$

$E(X): \quad$ The mean of random variable $X$

$\Lambda: \quad \Lambda=\lambda_{1}+\lambda_{2}+\cdots+\lambda_{n}$

$\mathfrak{R}(s)$ : The real part of complex number $s$

$\xi_{1}$ : $\quad$ The first operating time length of the system

$\eta_{1}: \quad \quad \quad \quad$ The first repair time length of the system

$P(Q): \quad$ The probability of event $Q$

$H(z),|z| \leq 1$ : The probability generating function of $H$. That is, $H(z)=\sum_{i=0}^{\infty} c_{i} z^{i}$.

\section{Acknowledgments}

The authors would like to thank the anonymous referees and editor for their valuable comments and suggestions. This work is supported by the Youth Foundation of Chongqing University of Technology of China (no. 2010ZQ14).

\section{References}

[1] J. H. Cao and Y. H. Wu, "Reliability analysis of a multistate system with a replaceable repair facility," Acta Mathematicae Applicatae Sinica, vol. 4, no. 2, pp. 113-121, 1988.

[2] K. Cheng, "Reliability analysis of a system in a randomly changing environment," Acta Mathematicae Applicatae Sinica, vol. 2, no. 3, pp. 219-228, 1985.

[3] J. H. Cao, "Availability and failure frequency of a Gnedenko system," Annals of Operations Research, vol. 24, no. 1-4, pp. 55-68, 1990.

[4] Y. Lam, "A note on the optimal replacement problem," Advances in Applied Probability, vol. 20, no. 2, pp. 479-482, 1988.

[5] J. Jia and S. Wu, "A replacement policy for a repairable system with its repairman having multiple vacations," Computers and Industrial Engineering, vol. 57, no. 1, pp. 156-160, 2009.

[6] J. Jia and S. Wu, "Optimizing replacement policy for a cold-standby system with waiting repair times," Applied Mathematics and Computation, vol. 214, no. 1, pp. 133-141, 2009.

[7] S. Wu and D. Clements-Croome, "Optimal maintenance policies under different operational schedules," IEEE Transaction on Reliability, vol. 54, no. 2, pp. 338-346, 2005. 
[8] Y. L. Zhang and G. J. Wang, "A deteriorating cold standby repairable system with priority in use," European Journal of Operational Reserch, vol. 183, no. 1, pp. 278-295, 2007.

[9] L. Yuan and J. Xu, "A deteriorating system with its repairman having multiple vacations," Applied Mathematics and Computation, vol. 217, no. 10, pp. 4980-4989, 2011.

[10] F. Rahmoune and D. Aissani, "Strong stability of queues with multiple vacation of the server," Stochastic Analysis and Applications, vol. 26, no. 3, pp. 665-678, 2008.

[11] D. R. Cox, "The analysis of non-Markovian stochastic processes by the inclusion of supplementary variables," Proceedings of the Cambridge Philosophical Society, vol. 51, pp. 433-441, 1955.

[12] W. Li, A. Alfa, and Y. Zhao, "Stochastic analysis of a repairable system with three units and two repair facilities," Microelectronics and Reliability, vol. 38, no. 4, pp. 585-595, 1998.

[13] R. B. Liu, Y. H. Tang, and C. Y. Luo, "A new kind of n-unit series repairable system and its reliability analysis," Mathematica Applicata, vol. 20, no. 1, pp. 164-170, 2007 (Chinese).

[14] R. B. Liu, Y. H. Tang, and B. S. Cao, "A new model for the $N$-unit series repairable system and its reliability analysis," Chinese Journal of Engineering Mathematics, vol. 25, no. 3, pp. 421-428, 2008 (Chinese).

[15] L. Guo, H. Xu, C. Gao, and G. Zhu, "Stability analysis of a new kind n-unit series repairable system," Applied Mathematical Modelling. Simulation and Computation for Engineering and Environmental Systems, vol. 35, no. 1, pp. 202-217, 2011.

[16] N. Tian and Z. G. Zhang, Vacation Queueing Models-Theory and Applications, Springer, New York, NY, USA, 2006.

[17] J. L. Schiff, The Laplace Transform: Theory and Applications, Springer, New York, NY, USA, 1999.

[18] R. E. Barlow and F. Proschan, Mathematical Theory of Reliability, John Wiley \& Sons, New York, NY, USA, 1965.

[19] J. H. Cao and K. Cheng, Introduction to Reliability Mathematics, Higher Education Press, Beijing, China, 1986. 


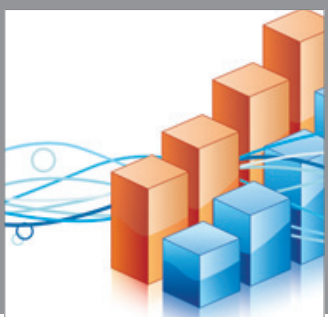

Advances in

Operations Research

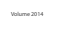

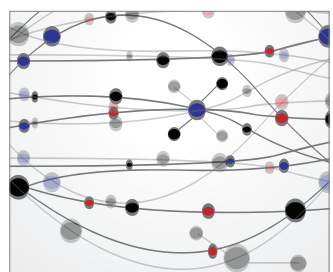

\section{The Scientific} World Journal
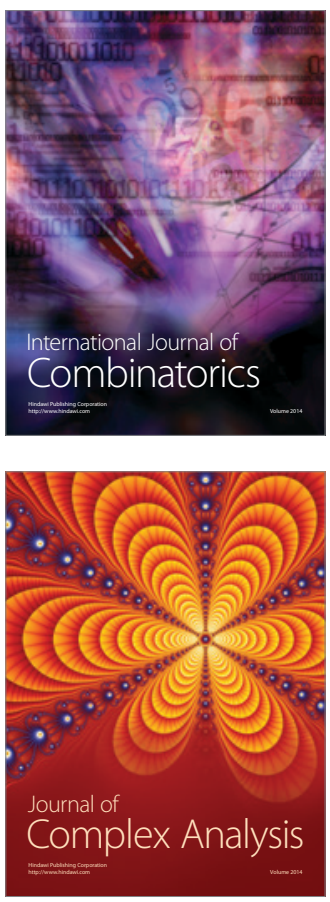

International Journal of

Mathematics and

Mathematical

Sciences
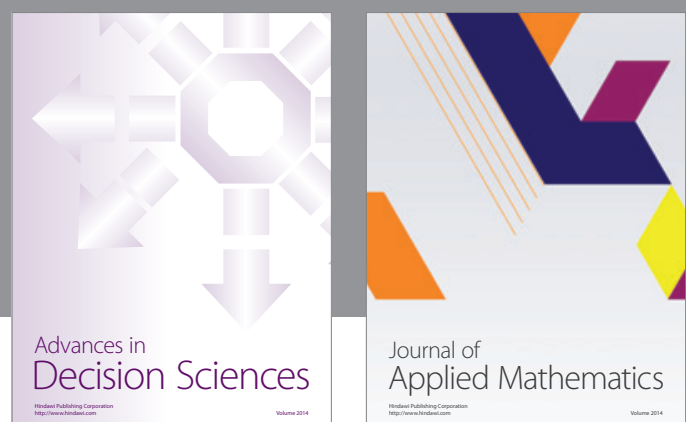

Journal of

Applied Mathematics
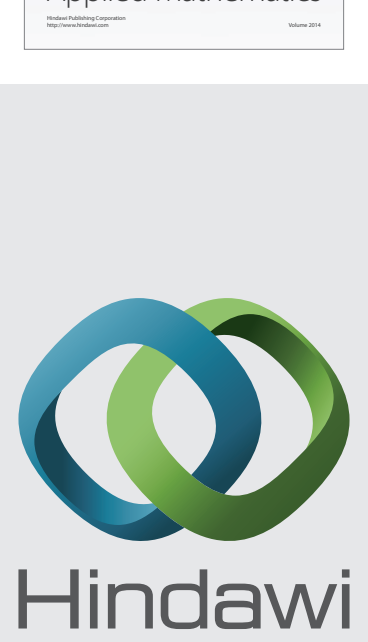

Submit your manuscripts at http://www.hindawi.com
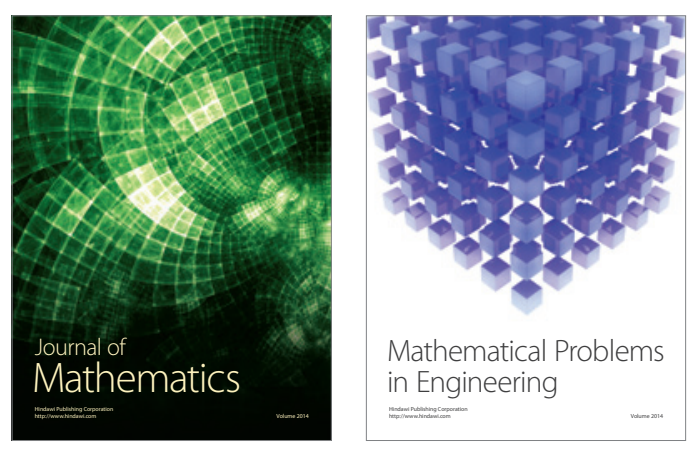

Mathematical Problems in Engineering
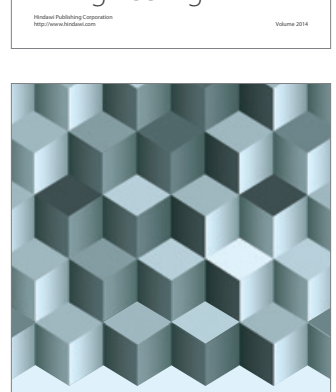

Journal of

Function Spaces
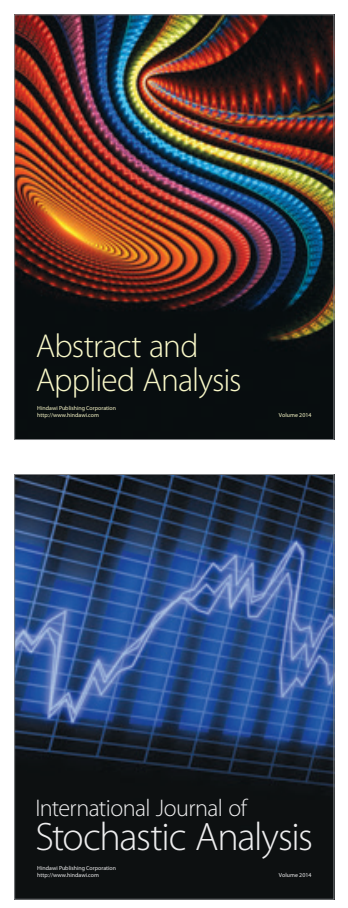

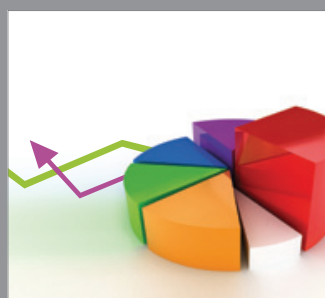

ournal of

Probability and Statistics

Promensencen
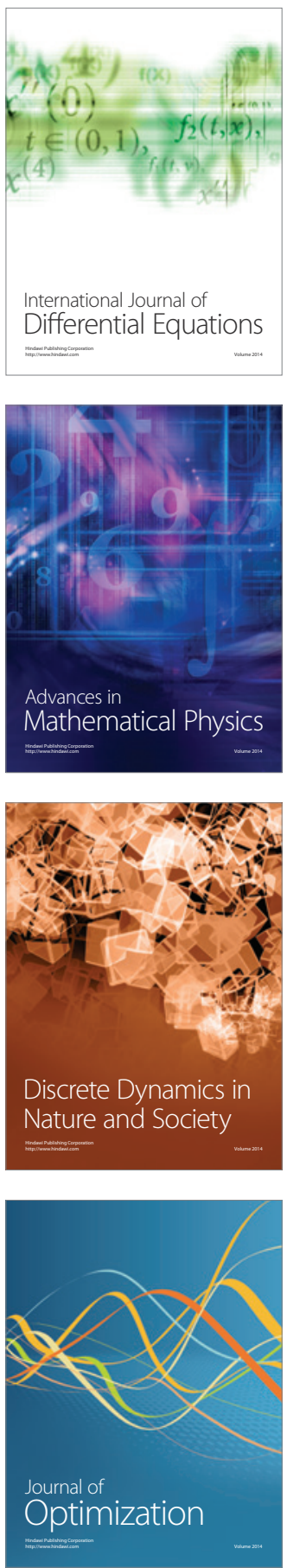